tions will always vary more or less. The only condition we have control over is treatment. It is doubtful if this bas practically varied in the above series - that is, so as to affect the results. It should be said that the average mortality of 12.6 per cent. does not correctly give the result of treatment in the Boston City Hospital, as the cases dying within a few days after admission bave not been excluded.

May it not well be that similar variations from chance alone may occur in statistics of mortality or statistics of the effect of special modes of treatment, of other diseases, such as diphtheria, pneumonia, chorea, epilepsy, etc.? At any rate until the contrary has been shown to be the case, it would seem that it is not safe to accept such statistics as evidence of the efficacy of treatment unless based upon the results in a very large number of cases. That so large a number as 800 to 1,000 is required in typhoid fever at least, is, I think, generally overlooked.

\section{CARDIAC STIMULANTS.}

BY HERMAN F. VIOKERY, M.D., BOSTON, MASS., Instructor in Clinical Medicine, Harvard University; Physician to Out-patients, Massachusetts General Hospital.

THE condition of the heart is constantly watched by the medical man. From time immemorial all physicians have sought in the pulse as exemplifying the heart of their patients, important aid with regard to diagnosis and weighty suggestions as to treatment. To-day no practitioner is too wise and none too simple to regard it. Neither the gravest consultation nor the most hurried and perfunctory visit is ended before skilful fingers, watched with a mixture of awe and approval by the family, have dwelt upon the radial artery. In all diseases at all stages the behavior of the heart is considered both as an index of the bodily state and a guide for the management of the case.

Unfortunately, this singular unanimity of the profession scarcely extends to the interpretation of the silent message which the heart vouchsafes, and still less to the mode of treatment appropriate to the occasion.

It is the object of the present paper to lay down some general rules as to the choice of cardiac remedies.

As has already been intimated, such a subject touches upon or overlaps all therapeutic measures in all conditions, so that our present consideration will unavoidably be very limited in its scope and incomplete in its details. Yet it is hoped that some practical benefit may be obtained.

In a general way, the disorders of the heart may be divided into those in which it shares in a general weakness of the system and those in which the original trouble is circulatory, using the word in its broadest sense.

The term general weakness is meant to include nervous debility. It is an interesting fact that the great majority of patients who come voluntarily to a physician because they believe the heart to be diseased are happily mistaken, and are really either dyspeptics, hy pochondriacs, sufferers from intercostal neuralgia or addicted to some excess which has occasioned palpitation. The hypochondriacs are many of them medical

Read, by invitation, at a meeting of the Plymouth District Medical Society, in Plymouth, July 17, 1895 . students, some of whom come in anxiety each year to their instructors for examination. I can recall only one of all such whom I have happened to auscult who actually had an organic lesion. The cases of intercostal neuralgia are apt to be anemic and to have tender spots along the course of an intercostal nerve and the heart is found to be normal. The victims of excess (in tea, coffee, tobacco or venery) are numerous. Their general appearance betrays them; and their heart-sounds have a hurried, quick, sharp, and as it were impatient character at the apex, unassociated with enlargement or persistent murmur. There is little muscular element; in the first sound. There may or may not be a slight basic systolic murmur, or at the apex a systolic whiff, once or twice during each respiratory cycle. The nervousness of the apex sounds is quite recognizable. The auscultatory signs are valuable in these functional cases, because the patient may not voluntarily confess his wrong-doing, and yet it is useless for him to swallow drugs without reforming his habits. It is better that for a time the special indulgence should be wholly stopped both because total abstinence is more likely to be attained than is temperance and because even a small amount of tobacco, for instance, will keep the already nicotized heart in its diseased state.

Alcoholic excess often affects the heart in the way just described, but the other bodily effects are usually too evident for one to need to auscult the heart in order to recognize the nature of the case, and besides arterial and myocardial degeneration may be present, so that the prognosis and treatment are not those of functional disturbance.

In the purely functional cases, bromide and a tonic usually bring immediate subjective improvement, and make it easier for the patient to abstain from the exciting cause. If there be anemia, iron is useful. Otherwise I am apt to prescribe compound tincture of cinchona. Nux vomica and strychnine are usually regarded as physiologically incompatible with the bromides, but in this class of cases ten or fifteen grains of bromide of sodium and five or ten minims of tincture of nux vomica seem to me an efficient combination. An analogous experience is the favorable effect upon some cases of enuresis when the belladona which has been used in vain is combined with nux vomica and the enuresis ceases. Clinical facts are sometimes like Wilkie Collins's heroine, "superior to logic."

Tincture of strophanthus in a dose rarely exceeding five minims, three times a day, has a good effect upon nervous palpitation.

Cases of general and nerrous debility sometimes have excessive cardiac weakness out of proportion to the other symptoms. The heart impresses one as thin and flabby. It may be somewhat dilated and there may be inconstant murmurs. The condition is not devoid of danger. There may be sudden collapse, with pulmonary congestion and death. At the same time the mind has considerable influence upon the disturbance, so that assurances that there is no valvular disease and that with returning bodily vigor the heart will become strong, contribute to the cure. About two years ago, a gentleman who had returned from a scientific expedition to Mexico with neurasthenia, and whose condition was causing his family much anxiety, allowed me to show him to a class of medical students as an unusually fine illustration of multiple inorganic murmurs. He recovered rather rapidly after a sea- 
voyage and is now well and actively engaged in his profession of civil engineer. For this neurasthenic state there is no specific remedy. Economy of nervous energy, and a cautious tonic regimen, with massage, graduated exercjse, naps, lunches, and such drugs as cod-liver oil, iron, arsenic and strychnine avail more than the ordinary cardiac tonics.

The specific fevers afford the greatest opportunity for careful observation, nice discrimination and good judgment in relation to the heart, of any bodily disorders other than circulatory. Some render the matter attractively simple by saying that fever contraindicates the use of digitalis. Others never allow any patient to get very near death before he swallows a certain amount of this panacea. Some of us believe the better way lies between these two extremes, but we are not above confessing that individual cases puzzle us.

It may be well to dwell somewhat upon the properties of digitalis itself, to learn what are the capabilities of the tool we desire to use. Here we find pretty general agreement that digitalis is a nervous stimulant, acting both on the heart itself and on the vagus nerve, and not directly supplying any energy but merely developing and regulating that which the heart already possesses. It is therefore plain that the drug if it leads to the display of more cardiac vigor, inasmuch as it does not contribute to the general -store, hastens the time when all energy will be exhausted and death ensue. We have an illustration of this fact in the result of the stimulation of the sympathetic nerve, which makes the heart beat stronger and more rapidly but also leads to earlier exhaustion than otherwise occurs, while a stimulation of the inhibitory fibres of the vagus in such a way as to slow and weaken the heart, actually enables that organ to store up energy.

Digitalis does more good when the heart is rapid, feeble, dilated, irregular in rhythm and especially when it is irregular in force. If such conditions develop in the course of any disease, we may expect the drug to bring some improvement. What little force there is will be more efficiently employed and a crisis may thus be tided over, and complete recovery ensue. In long continued fevers therefore, the longer such imperative indications for its employment are deferred and the shorter time the drug is given the more good it is likely to do. To prescribe it early in a long illness and where perhaps the only abnormalities in the heart are moderate weakness and a rapidity not disproportionately high as compared with the bodily temperature, will in most cases lead to nausea, malnutrition and early exhaustion rather than to any good. In this connection, might be called to mind the well-known difference in the average pulse-rate of pneumonia and of typhoid fever. In uncomplicated typhoid the heart-beats are lower relatively to the fever than in most diseases, so that if the pulse remains permanently at 120 it is of evil omen, while a rate of 140 in pneumonia is perfectly compatible with a favorable prognosis.

The books mention that digitalis does especial good in the exacerbations of chronic bronchitis, beyond what it usually accomplishes in general diseases. It has seemed to me that the explanation of this lies in the strain that long-continued bronchitis and consequent emphysema exert upon the right ventricle, so that mavy such patients are beart-cripples, and re- ceive the benefit from digitalis that dilated and enfeebled hearts usually get.

The habitual use of digitalis and other nervine cardiac stimulants in febrile diseases should in my opinion be deprecated. Many favorable cases can be guided to a successful issue without a single dose of the sort. The longer the exhibition of such drugs can be delayed, the less harm and the more good are they likely to do; yet, it is not impossible, although in my own limited experience it is rare, for them to save life, for example in the enfeebled state sometimes seen at the crisis of pneumonia, the subcutaneous injection of fifteen or twenty-five minims of tincture of digitalis may be of decided advantage.

In general systemic diseases, nux vomica or strychnine, alcohol and coffee commend themselves as more often useful and less often harmful than is digitalis. A temporary stimulation in collapse may be got from a hypodermic of atropine, one-sixtieth of a grain, or of cocaine, one-half a grain.

In the acute diseases of the heart cardiac sedatives, such as aconite, are sometimes preferable to stimulants. Every effort should be made to lessen the demands upon the affected but indispensable organ, by enforcing physical and mental rest, and by giving only easily digested nourishment in moderate amounts. If the circulation is being passably maintained, we should let well enough alone. The additional strain consequent upon the stimulation of digitalis and the like may magnify the inevitable damage done to the valves, aggravate the inflammation of the roughened pericardium, or exhaust the already impaired vitality of the muscular walls.

In chronic valvular disease, our first duty is to see that the demands made upon the enfeebled heart are not too great, or at least no greater than is absolutely necessary. If we can lessen the expenses of the economy so that they shall not exceed its diminished income, the heart may long continue to do a satisfactory business at the old stand. If, while it is thus carrying on the circulation, some one gives digitalis, not because it is needed but because there is a murmur, the heart may be prematurely exhausted. If, however, compensation has been ruptured, then digitalis may be of great benefit, tending to contract the dilated ventricles, and to strengthen and regulate the heart-beats.

All agree that mitral disease in its later stages is helped by digitalis; but, in regard to aortic regurgitation, many have an apprehension that digitalis may not be free from harm, because, the diastole being lengthened, more chance will exist for blood to regurgitate into an already overburdened left ventricle. In reply to this objection, we may say that digitalis tends to contract the left ventricle if dilated and also to invigorate it, and to render its contractions more efficient - advantages which more than counterbalance the lengthening of the diastole. One explanation why results are more unsatisfactory in cases of aortic regurgitation is that when once the heart begins to fail in this disease, life is near its termination and no drug, not even digitalis, can long defer death; whereas in mitral disease existence for years after the first symptoms of ruptured compensation is the rule rather than the exception.

An unfortunate characteristic of digitalis is that it contracts the arterioles and raises the intra-arterial tension, so that it is worse than folly to give it where 
the pulse is already of high tension and the aortic second sound loud and booming.

When there are symptoms of cardiac failure, when the apex cannot be located, the heart-sounds are feeble though without a murmur, and the radial artery is calcified, we know that the degenerated myocardium will not long respond to any stimulus. The rational line of treatment lies in the direction of lessening the heart's burdens, both by general measures and by the specific employment of nitro-glycerine to diminish arterial resistance ; and we may also prescribe potassic iodide in the hope that its absorbent powers may open up once more the calcified coronary arteries, and permit the better nourishment of the heart-muscle. Iron and arsenic may also contribute to nutrition. Strychnine and strophauthus often prove to be good stimulants. Alcohol is nothing but a palliative, and in this or any chronic cardiac disease is open to the grave suspicion of promoting degenerative processes. Against the exhibition of digitalis is its unfortunate property, just mentioned, of raising the intra-arterial tension. Given in combination with nitro-glycerine, we may hope to get the beneficial effects of both drugs. Abstract considerations aside, we do meet with cases of chronic myocarditis where nothing else gives the comfort which digitalis affords. It seems to search out and utilize every mite of energy the expiring organ still possesses. The subcutaneons employment of morphine is also sometimes valuable in the terminal stages of this and other forms of heart disease, not only giving relief but reprieving the patient from im. pending death.

Sparteine, adonidin, cactus grandiflora and convallaria majalis may in certain cases be useful. They rank far below the remedies already mentioned. With regard to the choice of a preparation of digitalis, I believe that the tincture is as efficient as the infusion, and that the apparent superiority of the infusion may very likely lie in the fact (pointed out recently by $\mathrm{H}$. C. Wood) that much stronger doses of the latter are given than of the former. In the present U. S. Phar. macopeia the infusion is made one-tenth the strength of the tincture, while its dose is said in the National Dispensatory to be not ten but twenty-six times larger. If we determine to try the drug in a given case, we should be prepared gradually to push the dose, if necessary, till either benefit or toxic symptoms are produced.

\section{Medical Mragrege.}

\section{REPORT ON PATHOLOGY.}

BY JAMES H. WRIGHT, M.D., Assistant in Pathology, Harvard Medical School.

THE LTIOLOGY OF THE SUPPURATIVE COMPLICATYONS OF TYPHOID FEVER.

THE question as to whether the typhoid bacillus is the causative agent in the production of the suppurative complications and sequelæ of typhoid fever has given rise to much discussion. Many observers hold to the view that the typhoid bacillus does cause these conditions, or in other words, can produce pus, for in certain cases it is the only organism found in the lesions. On the other hand, many bacteriologists consider, with Baumgarten, that these suppurations are merely the result of infection with the ordinary pyogenic cocci, as has been demonstrated by a number of investigators, and that in those cases in which the typhoid bacillus has been found, it was present only secondarily, while the true cause of the process, the pus-producing cocci, had accomplished their work and had died out.

'To this question there have recently appeared two interesting contributions. One of these is a case of multiple abscess formation in the kidneys in typhoid fever, reported by Flexner, $^{1}$ in which the most careful examination of the pus by all known methods failed to reveal the presence of any other organism than the typhoid bacillus. The other, by Dmochowski and Janowski, ${ }^{2}$ is a very exhaustive résumé of the literature of the subject, with an extensive experi. mental study of the results of inoculation with cultures of the typhoid bacillus.

These investigators have shown, by injecting the typhoid bacillus into the tissues of animals in a great variety of ways, that under certain conditions it is capable of producing suppuration, especially if the resistance of the tissue be diminished, as for instance by subjecting the tissues to the action of a weak emulsion of croton oil.

From a consideration of their work and the work of other observers who have studied this question, it would seem that while the typhoid bacillus may cause the suppurations of typhoid fover in a certain proportion of cases, yet in the majority of cases such processes are simply the result of secondary infection with either the staphylococcus pyogenes aureus or the streptococcus.

RIBBERT'S THEORY OF THE ORIGIN OF CARCINOMA.

In a recent number of Virchow's Archiv, Ribbert ${ }^{8}$ contributes some further observations in support of his theory that the origin of carcinoma is due to a primary new growth of connective tissue, which encroaches upon the epithelium of a part and separates portions of it from their normal relations, so that a growth of masses of epithelial cells arises, under ab. normal conditions. In the present paper he devotes particular attention to carcinomata of the alimentary canal, claiming to find in them the same evidences of a primary activity of connective-tissue elements of the mucosa as in the epidermoid cancers.

While this theory of Ribbert is plausible and interesting, it cannot be said that the evidence he has adduced in its favor is convincing.

EXPERIMENTAL NEPHRITIS FROM SODIUM CHLORIDE.

Levi administered to dogs and rabbits by the stom. ach and hypodermatically daily doses of one to four grammes per kilo of sodium chloride, and examined the kidneys microscopically after varying periods.

After 5 to 15 days there was found "granular necrosis" of the epithelium of the secreting tubules, while in the conducting tubules hyaline and granular casts, rich in nuclear fragments, were seen. The glomeruli also showed swelling and desquamation of their epithelium.

After 22 to 70 days, in dogs, more extensive lesions were present, consisting for the most part of marked "granular necrosis" with almost complete transfor-

1 Flexner : Journal of Pathology and Bacteriology, April, 1895.

2 Dmochowski and Janowski : Beiträge zur pathologischen Anatomie, E. Ziegler, Bd. xvii, Ht. 2 .

3 Ribbert: Virchow's Árchiv, Bd. oxll, Ht. 1

- Levi: Centralblatt für allgemeine Pathologie und pathologische Anatomie, Bd. vi, Nos. 12, 13 . 